Communications in Physics, Vol. 27, No. 3 (2017), pp. 267-276

DOI:10.15625/0868-3166/27/3/10611

\title{
RELAXATION RATE AND MOBILITY OF A TWO-DIMENSIONAL ELECTRON GAS IN MgZnO/ZnO HETEROSTRUCTURES INCLUDING EXCHANGE AND CORRELATION EFFECTS
}

\author{
VO VAN TAI ${ }^{a, b, \dagger}$ AND NGUYEN QUOC KHANH ${ }^{b}$ \\ ${ }^{a}$ Theoretical Physics Research Group, \\ Ton Duc Thang University, Ho Chi Minh City, Vietnam \\ ${ }^{b}$ University of Science - VNUHCM, \\ 227-Nguyen Van Cu Street, 5th District, Ho Chi Minh City, Vietnam \\ ${ }^{\dagger}$ E-mail: vovantai@tdt.edu.vn \\ Received 10 August 2017 \\ Accepted for publication 10 November 2017 \\ Published 19 November 2017
}

\begin{abstract}
We investigate the relaxation rate and mobility of a two-dimensional electron gas (2DEG) confined in $\mathrm{MgZnO} / \mathrm{ZnO}$ heterostructures (HSs) for temperaturesT $\leq 300 \mathrm{~K}$, taking into account exchange and correlation effects. We use the variational-subband-wave-function model for carrier confinement and assume that the electrons are confined to the lowest subband and scattered by acoustic phonons via deformation potential (DP) and piezoelectric (PE) fields, polar LO phonons, interface roughness (IRS), interface charges (IFCs) and the background impurities (BIs). The calculations are based on the linearized Boltzmann equation $(B E)$ and the relaxation time approximation, assuming the scattering by acoustic phonons to be quasi-elastic. We consider three physically distinct temperature ranges with respect to phonon scattering: the Bloch-Grüneisen $(B G)$, equipartition $(E P)$, and inelastic regimes. In the inelastic regime at high temperatures, where the scattering from polar $L O$ phonons becomes important, we solve directly the linearized $B E$ by an iterative method and compare the obtained results with those of the low-temperature and high-energy relaxation-time approximation. Our calculated low-temperature mobility is in good agreement with the recent experiment.
\end{abstract}

Keywords: $\mathrm{ZnO}$, heterostructure, scattering mechanism, correlation effect.

Classification numbers: 74.78.Fk; 79.60.Jv.

(C)2017 Vietnam Academy of Science and Technology 


\section{INTRODUCTION}

$\mathrm{ZnO}$, a direct wide band gap oxide semiconductor with würtzite structure has received great attention over the past years [1,2]. In particular, transport properties of the $2 \mathrm{DEG}$ in $\mathrm{MgZnO} / \mathrm{ZnO}$ HSs have been intensively studied for applications in electronic devices [3-8]. Gold $[9,10]$ has interpreted successfully mobility data $[11,12]$ at low temperatures by introducing charged-impurity and interface-roughness scattering taking into account the many-body and multiple scattering effects. Tsaousidou has calculated numerically the acoustic-phonon limited mobility and obtained the mobility limited by elastic scattering from the experimental data at $T=0.3 K$ [13]. Following the treatment of Kawamura and Das Sarma for AlGaAs/GaAs HSs [14], Begum and coworkers [15] have solved the linearized BE using iterative technique and calculated the mobility limited by the inelastic polar LO phonon and quasi-elastic acoustic (AC) phonons in the EP regime along with the IFCs, IFR and BIs for $\mathrm{MgZnO} / \mathrm{ZnO} \mathrm{HSs}$. They have treated the effects of screening of the 2DEG within the random-phase approximation (RPA) without a finite local-field correction (LFC) describing the exchange-correlation effects beyond the RPA and compared their results with the experimental data at low temperatures [16]. However, the calculations in the EP regime are valid only at high temperatures, when phonon energy is negligibly small compared with the thermal energy of electrons [14], and the LFC is very important for a 2DEG in $\mathrm{MgZnO} / \mathrm{ZnO}$ HSs because the interaction parameter $r_{s}$ is much larger compared to the one in $\mathrm{AlGaAs} / \mathrm{GaAs}$ heterostructures due to the larger effective mass in $\mathrm{ZnO}$ based materials [9-10,13]. Li and coworkers [17] have calculated the mobility of $2 \mathrm{DEG}$ in $\mathrm{ZnMgO} / \mathrm{ZnO} \mathrm{HSs}$ in the $\mathrm{BG}$ regime, when the acoustic phonon energy is comparable to the thermal energy of electrons, taking into account exchange and correlation effects. They have, however, considered only the temperature dependence using one form of the LFC, and have neglected the LO phonon contribution to the total mobility. In this paper, we apply the calculation of Kawamura and Das Sarma [14] to a 2DEG in MgZnO/ZnO HSs considering three physically distinct temperature ranges with respect to phonon scattering: the BG, EP, and inelastic regimes. We take into account exchange and correlation effects using three LFC models, and study both temperature and density dependence of relaxation rate and mobility. Unlike above mentioned papers $[9,10,13,15,17]$, we present our theoretical results in more detail for convenient use in future calculations and interpretations of experimental data.

\section{THEORY}

\section{II.1. Boltzmann transport equation}

We consider a 2DEG confined spatially along the direction perpendicular to the interface in $\mathrm{MgZnO} / \mathrm{ZnO} \mathrm{HSs}$, and assume that the electrons can be described by Fang-Howard variational wave function and occupy only the lowest subband [13-15].

For low electric fields, using the principle of detailed balance, we get the following linearized BE [14],

$$
1=\frac{A}{(2 \pi)^{2}} \int d k^{\prime} \frac{1-f_{0}\left(E^{\prime}\right)}{1-f_{0}(E)}\left[\varphi(E)-\frac{k^{\prime} \cos \theta}{k} \varphi\left(E^{\prime}\right)\right] \frac{S\left(k, k^{\prime}\right)}{\varepsilon^{2}(q, T)},
$$

where $A$ denotes the area of the plane $(x y), S\left(k, k^{\prime}\right)$ is the differential scattering rate from state $k$ to state $k^{\prime}, \theta$ is the angle between $k$ and $k^{\prime}, f_{0}(E)$ is the equilibrium Fermi-Dirac distribution function, $\varphi(E)$ is the perturbation distribution function and $\varepsilon(q, T)$ is the 2DEG dielectric function. In the 
RPA, $\varepsilon(q, T)$ is given as $[14,18]$

$$
\varepsilon(q, T)=1+\frac{2 \pi e^{2}}{\kappa_{w}} \frac{1}{q} F_{\mathrm{C}}(q)[1-G(q)] \Pi(q, T),
$$

where is $\kappa_{w}$ the static dielectric constant, $G(q)$ is the LFC describing the exchange-correlation effects beyond the RPA, $\Pi(q, T)$ is the polarizability of $2 \mathrm{DEG}$ and $F_{\mathrm{C}}(q)$ is the form factor associated with the subband wave function [14].

The differential scattering rate $S\left(k, k^{\prime}\right)$ depends on scattering mechanism via perturbing Hamiltonian $H_{I}$ and has the form [21]

$$
S\left(k, k^{\prime}\right)=\frac{2 \pi}{\hbar}\left|\left\langle k^{\prime}\left|H_{I}\right| k\right\rangle\right|^{2} \delta\left(E^{\prime}-E\right),
$$

for elastic scattering and

$$
S\left(k, k^{\prime}\right)=\frac{2 \pi}{\hbar}\left|\left\langle k^{\prime}, n_{Q}^{\prime}\left|H_{I}\right| k, n_{Q}\right\rangle\right|^{2} \delta\left(E^{\prime}-E \pm \hbar \omega_{Q}\right),
$$

for phonon scattering. Here $Q=\left(q, q_{z}\right)$ and $\hbar \omega_{Q}$ is the wave-vector and the energy of 3D phonons, respectively.

\section{II.2. Relaxation time in the elastic regime}

For elastic scattering processes such as IFCs, IFR and BIs, the relaxation time is given by $[18,20]$

$$
\frac{1}{\tau_{t}(E)}=\frac{m^{*}}{\pi \hbar^{3}} \int_{0}^{\pi} d \theta(1-\cos \theta) \frac{\left\langle\left|U_{j}(q)\right|^{2}\right\rangle}{\varepsilon^{2}(q, T)},
$$

where $\left\langle\left|U_{j}(q)\right|^{2}\right\rangle$ is the random potential which depends on the scattering mechanism. For IFCs, IFR and BIs the corresponding random potentials are given in Refs. [22-24].

Assuming that acoustic phonons do not carry away much energy we can use the quasielastic approximation and obtain the following closed-form forms for $\left\langle\left|U_{j}(q)\right|^{2}\right\rangle$ in the BG regime [14]

$$
\left\langle\left|U_{j}^{B G}(q)\right|^{2}\right\rangle=\frac{1}{\pi} \int_{0}^{+\infty} d q_{z} \frac{\left|C_{j}\left(q, q_{z}\right)\right|^{2}}{\varepsilon^{2}(q, T)}\left|I\left(q_{z}\right)\right|^{2} \Delta(E) .
$$

Here,

$$
\Delta(E)=\left\{n_{Q}\left[1-f_{0}\left(E+\hbar \omega_{Q}\right)\right]+\left(n_{Q}+1\right)\left[1-f_{0}\left(E-\hbar \omega_{Q}\right)\right]\right\} /\left[1-f_{0}(E)\right],
$$

where $n_{Q}$ is the Bose factor, $I\left(q_{z}\right)$ is the overlap integral for intraband scattering [14], and $C_{j}\left(q, q_{z}\right)$ are given by

$$
\left|C_{\mathrm{DP}}(Q)\right|^{2}=\frac{\Xi_{\mathrm{DP}}^{2} \hbar Q}{2 \rho v_{l}}=\frac{\Xi_{\mathrm{DP}}^{2} \hbar \sqrt{q^{2}+q_{\mathrm{Z}}^{2}}}{2 \rho v_{l}}
$$

for the deformation potential and

$$
\begin{aligned}
\left|C_{P E l}(Q)\right|^{2} & =\frac{e^{2} \hbar}{2 \rho v_{l}} \frac{1}{Q} H_{l}(Q), \\
\left|C_{P E t}(Q)\right|^{2} & =\frac{e^{2} \hbar}{2 \rho v_{t}} \frac{1}{Q} H_{t}(Q),
\end{aligned}
$$


for the longitudinal PE and transverse PE-coupled scattering, where

$$
\begin{aligned}
& H_{l}\left(q, q_{\mathrm{z}}\right)=\frac{q_{\mathrm{z}}^{2}}{q^{2}+q_{\mathrm{z}}^{2}}\left[h_{33}-h_{a} \frac{q^{2}}{q^{2}+q_{\mathrm{z}}^{2}}\right]^{2}, \\
& H_{t}\left(q, q_{\mathrm{z}}\right)=\frac{q^{2}}{q^{2}+q_{\mathrm{z}}^{2}}\left[h_{15}+h_{a} \frac{q_{\mathrm{z}}^{2}}{q^{2}+q_{\mathrm{z}}^{2}}\right]^{2},
\end{aligned}
$$

with $h_{a}=h_{33}-h_{b} ; h_{b}=h_{13}+2 h_{15}, h_{i j}$ being the components of the piezoelectric tensor [25]. Here $\Xi_{D P}$ is DP constant, $\rho$ is the mass density, and $v_{l}\left(v_{t}\right)$ is the longitudinal (transverse) velocity of sound in $\mathrm{ZnO}$.

In the EP regime we have $\hbar \omega_{Q} /\left(k_{B} T\right) \ll 1$ so that $n_{Q} \approx n_{Q}+1 \approx k_{B} T /\left(\hbar \omega_{Q}\right)>>1$ and the random potentials reduce to the following forms

$$
\begin{aligned}
\left\langle\left|U_{D P}^{E P}(q)\right|^{2}\right\rangle & =\frac{3 \Xi_{\mathrm{DP}}^{2} b k_{B} T}{16 \rho v_{l}^{2}}, \\
\left\langle\left|U_{P E l}^{E P}(q)\right|^{2}\right\rangle & =\frac{e^{2} k_{B} T}{2 \rho v_{l}^{2}} \frac{1}{q} f_{l}(w), \\
\left\langle\left|U_{P E t}^{E P}(q)\right|^{2}\right\rangle & =\frac{e^{2} k_{B} T}{2 \rho v_{t}^{2}} \frac{1}{q} f_{t}(w) .
\end{aligned}
$$

Here $f_{l}\left(f_{t}\right)$ are the dimensionless form factors for the longitudinal (transverse) acousticphonon modes associated with the piezoelectric coupling defined by

$$
\begin{aligned}
& f_{l}(w)=\frac{h_{a}^{2}}{48}\left(15 f_{0}-33 f_{1}+12 f_{2}-f_{3}\right)+\frac{h_{a} h_{b}}{4}\left(3 f_{0}-5 f_{1}+f_{2}\right)+\frac{h_{b}^{2}}{2}\left(f_{0}-f_{1}\right), \\
& f_{t}(w)=\frac{h_{a}^{2}}{48}\left(3 f_{0}+3 f_{1}-6 f_{2}+f_{3}\right)+\frac{h_{15} h_{a}}{4}\left(f_{0}+f_{1}-f_{2}\right)+\frac{h_{15}^{2}}{2}\left(f_{0}+f_{1}\right),
\end{aligned}
$$

where $w=q / b$ and $f_{0}, f_{1}, f_{2}, f_{3}$ are given in [25].

\section{II.3. Transport in the inelastic regime}

As the temperature of the $2 \mathrm{DEG}$ is raised, the scattering from polar LO phonons starts to dominate the transport properties of the 2DEG. The previous simplifications in the evaluation of the perturbation distribution associated with the quasi-elastic approximation are no longer valid and the linearized BE must be solved directly. The 2D linearized BE (1) can be written in the form of a difference equation coupling $\varphi(E)$ with $\varphi\left(E \pm \hbar \omega_{0}\right)$,

$$
1=S_{0}(E) \varphi(E)-S_{a}(E) \varphi\left(E+\hbar \omega_{0}\right)-S_{e}(E) \varphi\left(E-\hbar \omega_{0}\right),
$$

where, $S_{o}(E), S_{a}(E)$ and $S_{e}(E)$ are given in [14].

Although no closed-form expressions exists for $\varphi(E)$, a relaxation time may be obtained under certain approximations [14]. For low enough temperatures,

$$
\tau_{T L}(E)=S_{0}^{-1}(E) .
$$

and for higher temperatures,

$$
\tau_{H E}(E)=1 /\left[S_{0}(E)-S_{a}(E)-S_{e}(E)\right] .
$$


Once the values of $\varphi(E)$ are obtained, the mobility can be calculated from $\mu=e\left\langle\tau_{t}(E)\right\rangle / m^{*}$, with $\tau_{t}(E)$ replaced by $\varphi(E)$ and the total mobility are included by the Matthiessen's rule [13-15].

\section{RESULTS AND DISCUSSION}

In this section, we present our numerical calculations of the relaxation time $\tau_{t}(E)$ and the mobility $\mu$ for parameters characteristic of $\mathrm{ZnO}[15,16]: m^{*}=0.29 m_{e}, \rho=5.67 \mathrm{~g} / \mathrm{cm}^{3}$, $v_{l}=6.365 \times 10^{5} \mathrm{~cm} / \mathrm{s}, v_{t}=2.735 \times 10^{5} \mathrm{~cm} / \mathrm{s}, \Xi_{D P}=15 \mathrm{eV}, \kappa_{w}=8.45, \kappa_{\infty}=4.1, h_{13}=-5.683 \times$ $10^{7} \mathrm{~V} / \mathrm{cm}, h_{15}=-5.108 \times 10^{7} \mathrm{~V} / \mathrm{cm}, h_{33}=1.277 \times 10^{8} \mathrm{~V} / \mathrm{cm}$, and $\hbar \omega_{0}=72 \mathrm{meV}$. Gold and Tsaousidou $[9,10,13]$ have shown that many-body effects are very important for 2DEGs in $\mathrm{MgZnO} / \mathrm{ZnO} \mathrm{HSs}$ due to large interaction parameter $r_{s}$. Therefore, we take into account exchange and correlation effects using a LFC $G(q)$. In the Hubbard approximation, only exchange effects are taken into account and the $\mathrm{LFC}$ has the form $G_{\mathrm{H}}(q)=q /\left(2 \sqrt{q^{2}+k_{\mathrm{F}}^{2}}\right)[9,10,18]$. We also use analytical expressions of the LFC $\left(G_{G A}\right)$ according to the numerical results obtained in Ref. [19] where both exchange and correlation effects are taken into account

$$
G_{G A}(q)=r_{s}^{4 / 3} \frac{1.402 q}{\sqrt{2.644 C_{1}^{2} q_{s}^{2}+C_{2}^{2} r_{s}^{4 / 3} q^{2}-C_{3} r_{s}^{2 / 3} q_{s} q}},
$$

with $q_{s}=2 / a^{*}, a^{*}=\hbar^{2} \kappa_{w} /\left(m^{*} e^{2}\right)$ is Bohr radius and $r_{s}=1 / \sqrt{N_{s} \pi\left(a^{*}\right)^{2}}$ is the Wigner-Seitz parameter; the three coefficients $C_{i}(i=1,2,3)$ are quoted from Ref. [19]. In the following, we use a vanishing depletion density $N_{d e p l}=0$ as in Ref. [9].

\section{III.1. Relaxation rate and mobility due to scattering from AC phonons}

Using the quasi-elastic approximation for acoustic phonons, we have calculated the energy dependence of the EP and BG relaxation rates for the DP and PE phonons in different $G(q)$ models. The results shown in figures 1 and 2 indicate that the EP and BG relaxation rates are similar at high temperatures as expected and the exchange and correlation effects are significant.

To assess the validity of the EP approximation we show in Fig. 3 the temperature dependence of the BG (solid lines) and EP (dashed lines) mobility $\mu$ due to acoustic phonons at $N_{s}=6.8 \times 10^{10} \mathrm{~cm}^{-2}$ and $1.7 \times 10^{11} \mathrm{~cm}^{-2}$ for different $G(q)$ models. We see that the EP approximation underestimates the mobility at low temperatures since EP tends to overestimate the Bose factor for acoustic phonons. The transition into the $\mathrm{BG}$ regime happens at a higher temperature for higher densities, in accordance with the rough estimates $k_{B} T_{B G} \approx 2 k_{F} \hbar v_{l}$ given in [14]. The exchange and correlation effects on the mobility are considerable at low temperatures and become less important at high temperature.

\section{III.2. Mobility in the inelastic regime}

In order to understand the importance of the $\mathrm{LO}$ phonon scattering, we have solved the $\mathrm{BE}$ using the iteration technique. Based on obtained perturbation function $\varphi^{-1}(E)$, low-temperature relaxation rate $\tau_{L T}^{-1}(E)$ and high-energy relaxation rate $\tau_{H E}^{-1}(E)$, we have calculated the reciprocal phonon limited mobility. In Fig. 4 we show a comparison of the mobility calculated via the iterative scheme with that calculated from the closed-form relaxation rate approximations. It is seen from the figure that at room temperature the low-temperature relaxation rate $\tau_{L T}^{-1}(E)$ underestimates and the high-energy relaxation rate $\tau_{H E}^{-1}(E)$ overestimates the true mobility. However, in 

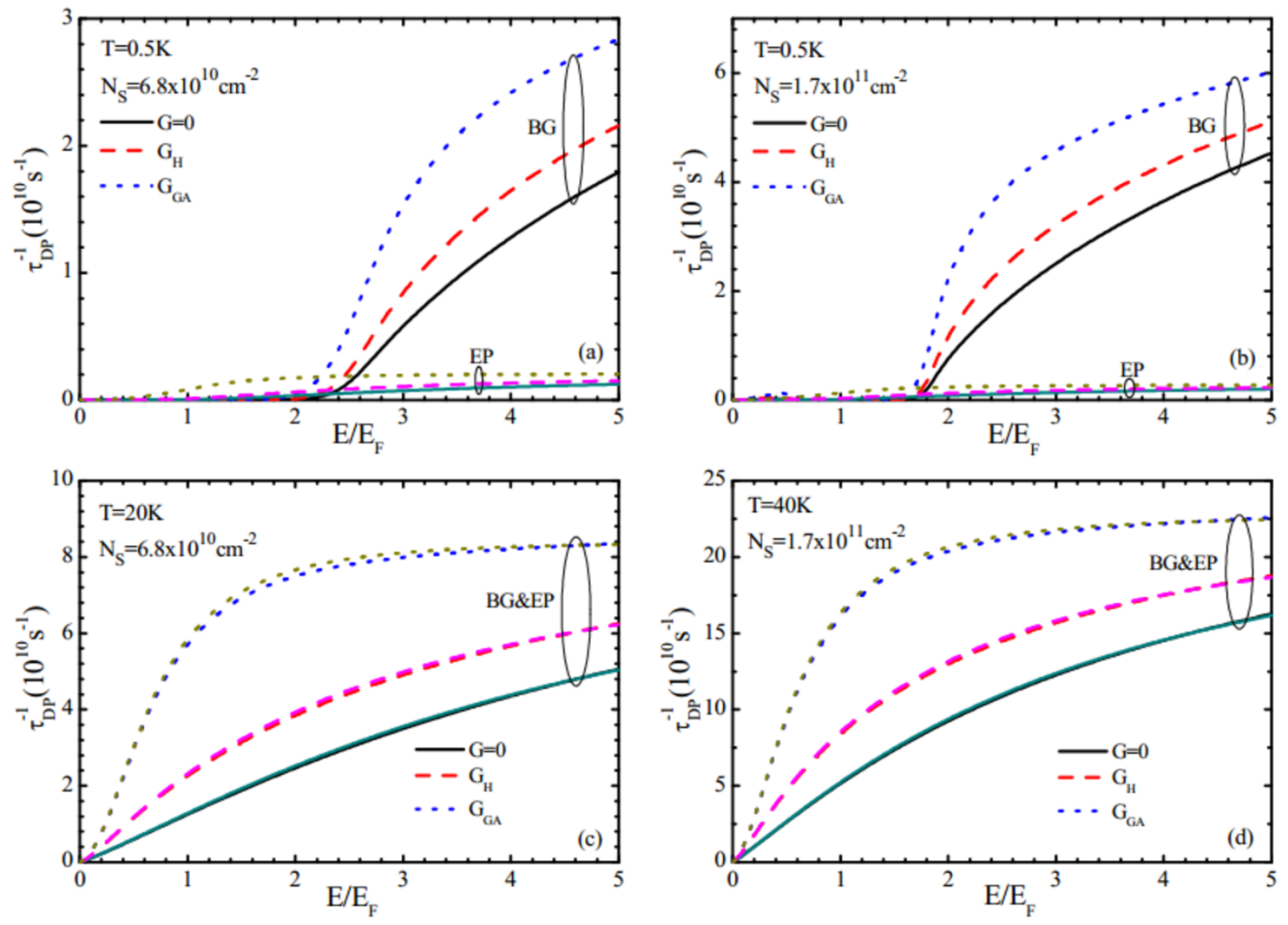

Fig. 1. Energy dependence of the EP and BG relaxation rates due to the DP phonons for two values of carrier density at two temperatures in different $G(q)$ models.

contrary to the case of AlGaAs/GaAs HSs [14], the deviation of $\tau_{H E}^{-1}(E)$ from the iterative value is much larger than that of $\tau_{L T}^{-1}(E)$. We also observe that the scattering from LO phonons is important only for $T>150 \mathrm{~K}$ when the total mobility $\mu$ differs notably from $\mu_{a c}$.

\section{III.3. Mobility due to the scattering by phonons and defects}

To find the key scattering mechanism limiting the mobility of real samples, we show in Fig. 5 the variation of the total mobility $\mu$ and individual contributions, calculated in the RPA, with electron concentration assuming electrons to be scattered by inelastic polar LO phonons, DP phonons, PE phonons, IFR, BIs and IFCs. We see that at very low temperatures the IFR determines the mobility for high density and at room temperature the polar LO phonon scattering dominates for all concentrations considered. The density dependence of the total mobility is stronger at lower temperatures.

In figures 6 and 7 we show the total mobility $\mu$ and individual contributions versus temperature for two values of electron densitywith all many-body effects included. We compare our calculations with the recent available experimental data of Falson et al. [16]. Note that, from above discussion, the LO phonon contribution is negligible for $T<20 \mathrm{~K}$, only acoustic phonons are taken into account in figures 6 and 7 . We see that our results for the total mobility calculated 

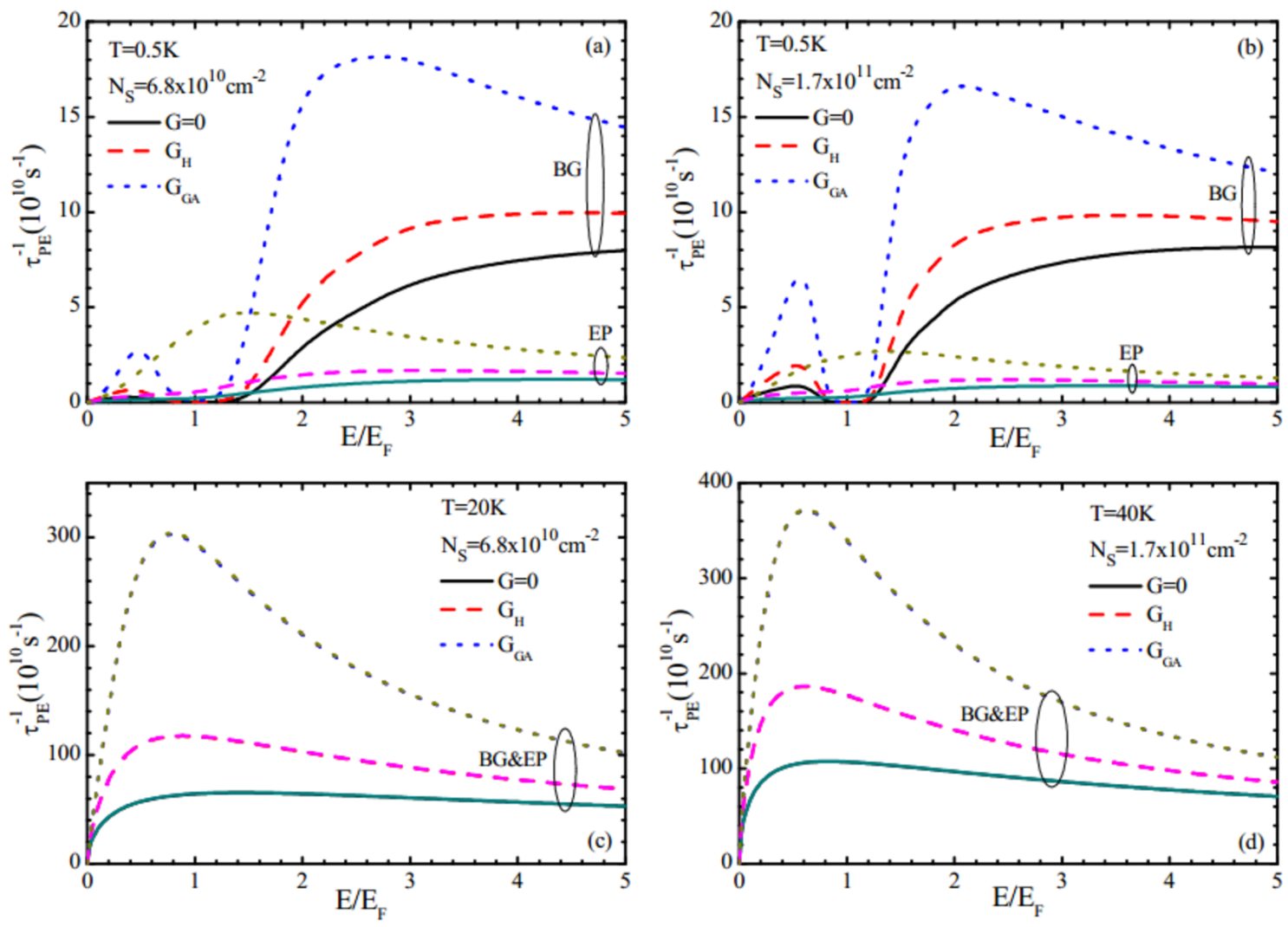

Fig. 2. Energy dependence of the EP and BG relaxation rates due to the PE phonons for two values of carrier density at two temperatures in different $G(q)$ models.
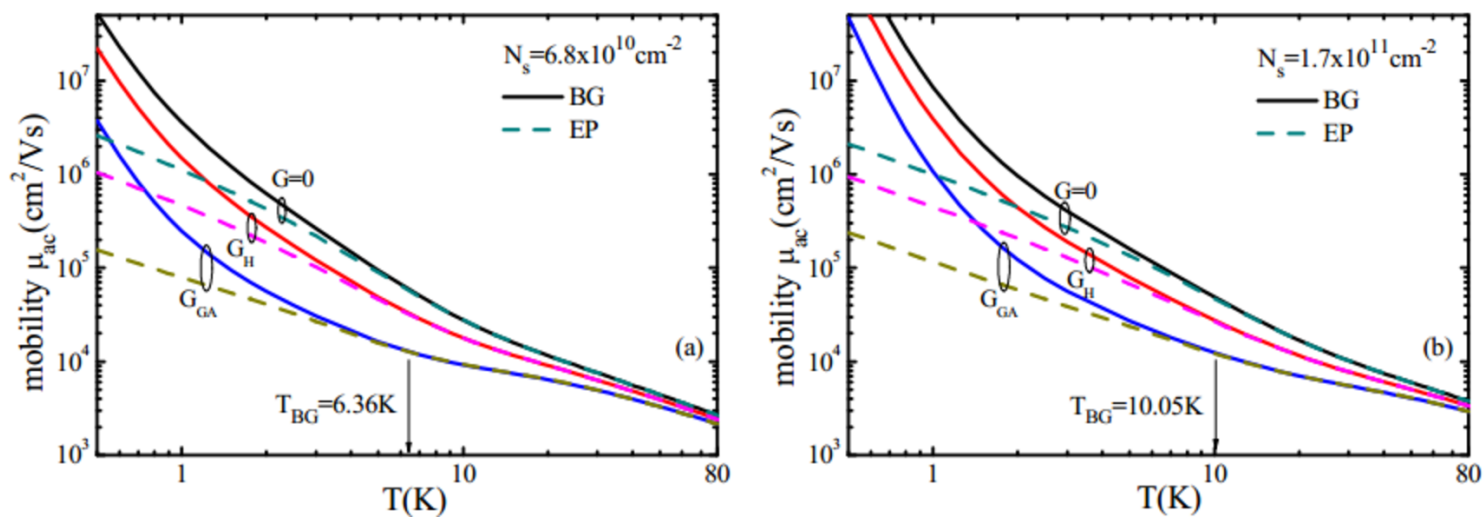

Fig. 3. Temperature dependence of the BG (solid lines) and EP (dashed lines) mobility $\mu$ due to acoustic phonons for different $G(q)$ models.

from the full form for the relaxation rates including many-body effects (solid lines denoted by Total12) are in good agreement with the experimental data [16] for temperature range and electron 

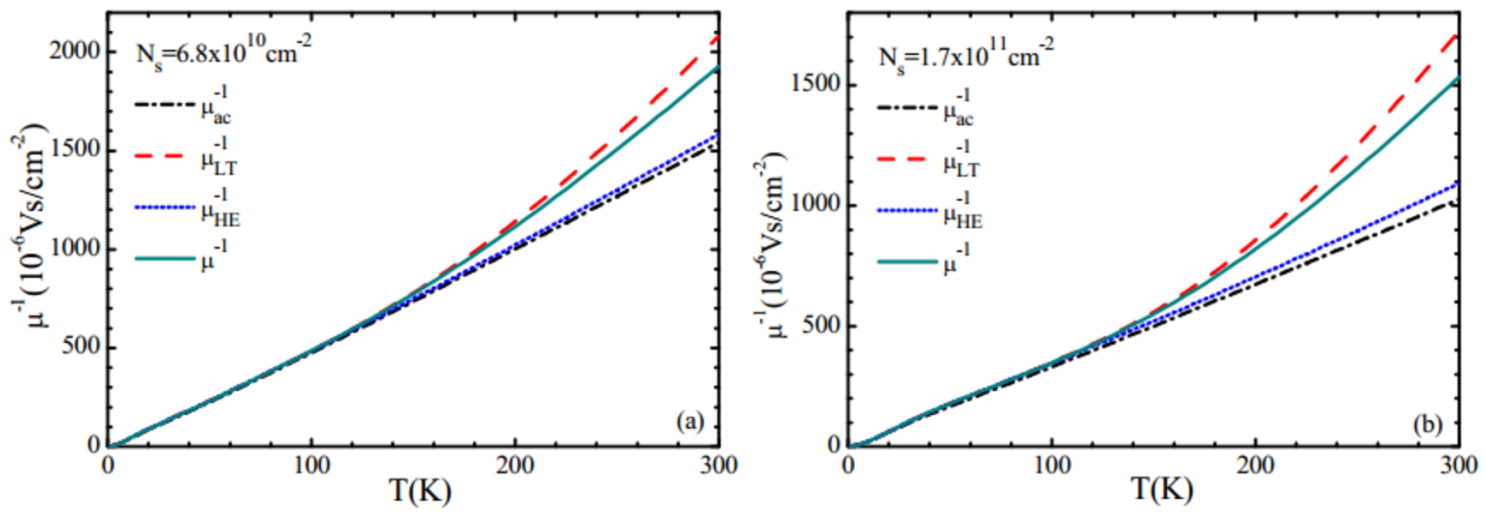

Fig. 4. The reciprocal mobility limited by acoustic and polar LO phonons (solid line) and approximations based on the low-temperature relaxation rate $\tau_{L T}^{-1}(E)$ (dashed line) and the high-energy relaxation rate $\tau_{H E}^{-1}(E)$ (dotted line). The dashed-dotted line is the reciprocal acoustic-phonon limited mobility $\tau_{a c}^{-1}(E)$.
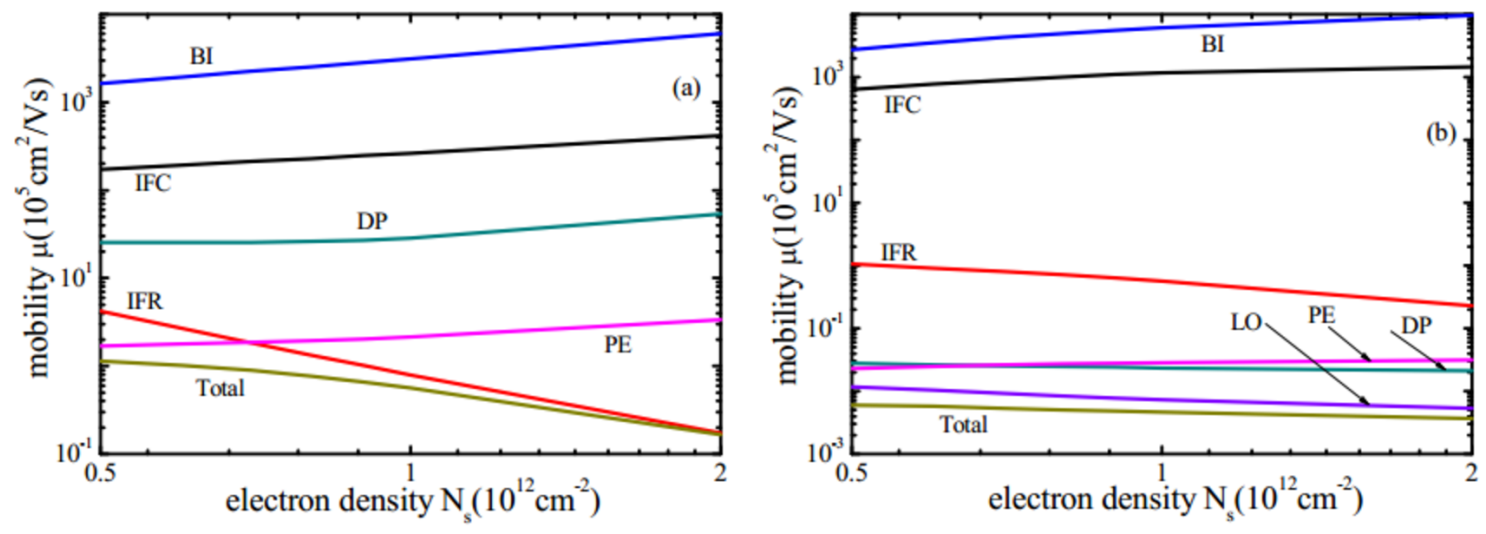

Fig. 5. Variation of the total mobility $\mu$ and individual contributions vs electron concentration with $G(q)=0 ; \Delta=2.2 \AA ; \Lambda=22 \AA ; N_{B 1}=10^{13} \mathrm{~cm}^{-3} ; N_{B 2}=10^{12} \mathrm{~cm}^{-3}$; $N_{I F C}=5 \times 10^{7} \mathrm{~cm}^{-2}$ for (a) $T=6 \mathrm{~K}$ and (b) $T=300 \mathrm{~K}$.

concentrations considered. The EP approximation (the lines denoted by Total13) underestimates the total mobility at low temperatures as expected. Note that the IRS parameters, interface impurity density $N_{I F C}$ and background doping levels $N_{B 1}, N_{B 2}$ were chosen to fit approximately the experimental data given in Ref. [16].

\section{CONCLUSION}

In summary, we have investigated the transport properties of the 2DEG in $\mathrm{MgZnO} / \mathrm{ZnO}$ HSs considering elastic scattering from IRS, IFCs and BIs, quasi-elastic scattering from DP and PE-coupled acoustic phonons, and the inelastic scattering from the polar LO phonons. The linearized $\mathrm{BE}$ is solved using the relaxation time approximation for acoustic phonons and the iteration method for LO polar phonons. We have shown that the BG relaxation rates and mobility 

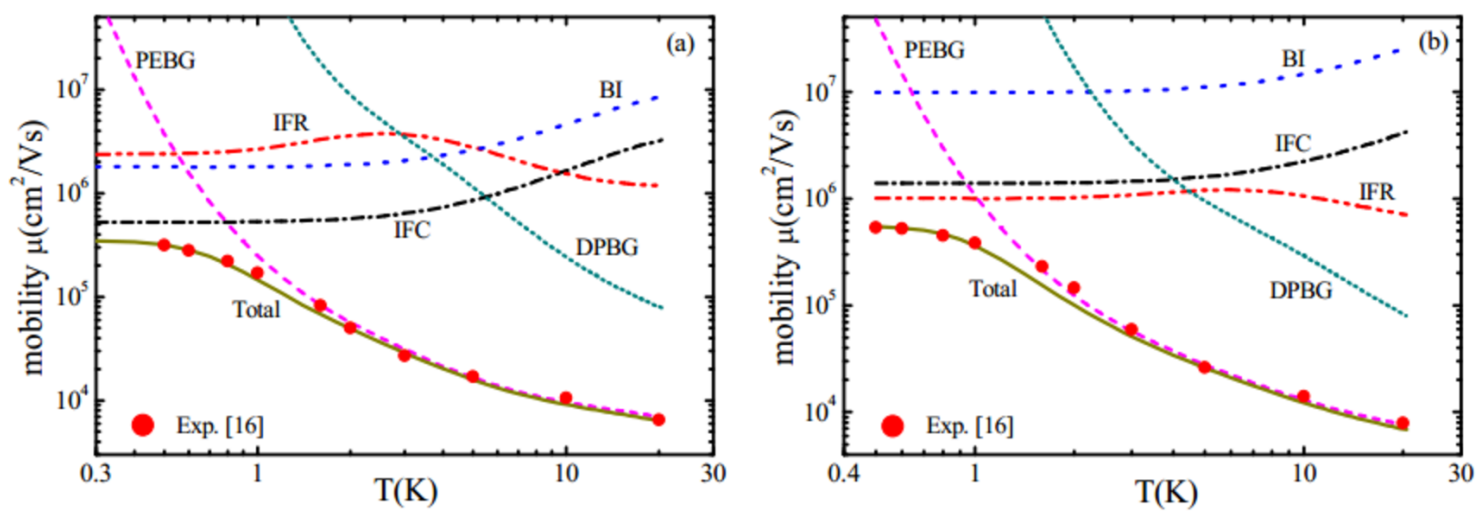

Fig. 6. Variation of the total mobility $\mu$ and individual contributions vs temperature with $G(q)=G_{G A}(q), N_{I F C}=5 \times 10^{7} \mathrm{~cm}^{-2}, N_{B 1}=10^{13} \mathrm{~cm}^{-3}$ for (a) $N_{s}=6.8 \times 10^{10} \mathrm{~cm}^{-2}$, $\Delta=2.8 \AA, \Lambda=30 \AA, N_{B 2}=2 \times 10^{12} \mathrm{~cm}^{-3}$ and (b) $N_{s}=1.7 \times 10^{11} \mathrm{~cm}^{-2}, \Delta=2.2 \AA$, $\Lambda=22 \AA, N_{B 2}=10^{12} \mathrm{~cm}^{-3}$. The solid dots represent the experimental results of Ref. [16].
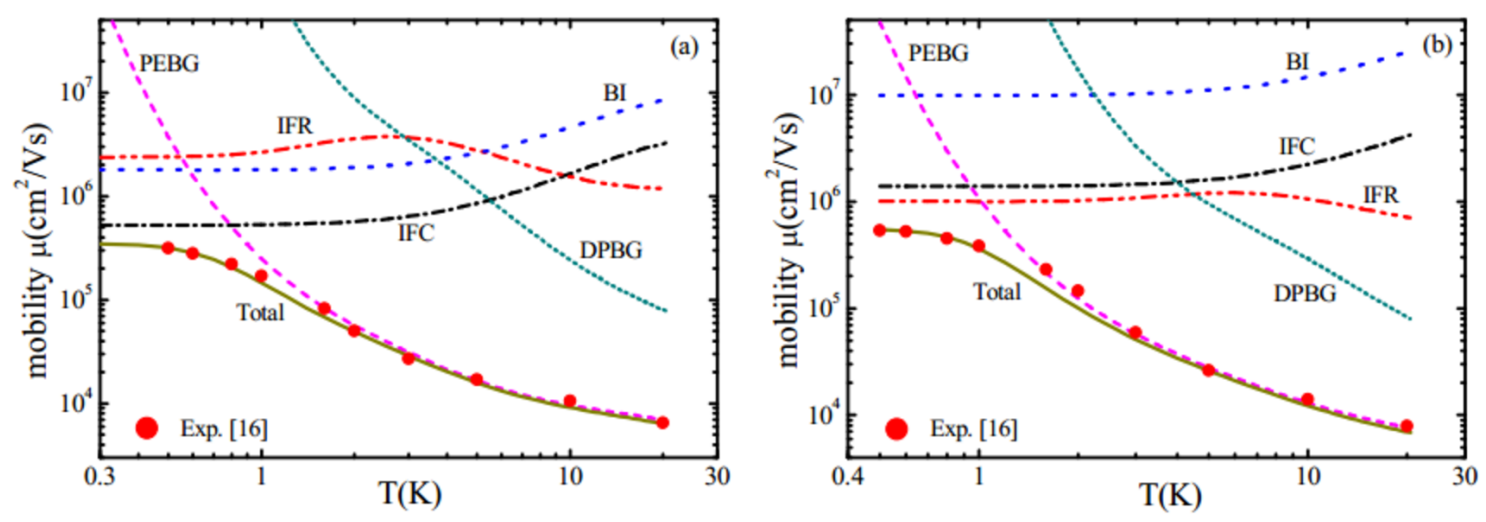

Fig. 7. Variation of the total mobility $\mu$ and individual contributions vs temperature with $G(q)=G_{G A}(q), N_{I F C}=5 \times 10^{7} \mathrm{~cm}^{-2}$, for four samples: (a) $N_{s}=2 \times 10^{12} \mathrm{~cm}^{-2}, \Delta=2 \AA$, $\Lambda=15 \AA, N_{B 1}=10^{14} \mathrm{~cm}^{-3} \quad N_{B 2}=10^{13} \mathrm{~cm}^{-3}$; (b) $N_{s}=7 \times 10^{11} \mathrm{~cm}^{-2}, \Delta=2.2 \AA$, $\Lambda=22 \stackrel{o}{A}, N_{B 1}=7 \times 10^{13} \mathrm{~cm}^{-3} \quad N_{B 2}=10^{13} \mathrm{~cm}^{-3}$; (c) $N_{s}=4.5 \times 10^{11} \mathrm{~cm}^{-2}, \Delta=2.4 \stackrel{o}{A}$, $\Lambda=21 \AA, N_{B 1}=5 \times 10^{13} \mathrm{~cm}^{-3} \quad N_{B 2}=5 \times 10^{12} \mathrm{~cm}^{-3}$ and (d) $N_{s}=1.4 \times 10^{11} \mathrm{~cm}^{-2}$, $\Delta=2 \AA, \Lambda=15 \AA, N_{B 1}=10^{13} \mathrm{~cm}^{-3} \quad N_{B 2}=10^{12} \mathrm{~cm}^{-3}$. The solid dots represent the experimental results of Ref. [16].

are similar to those calculated using the EP approximation at high temperatures as expected. It is found that the exchange and correlation effects on the relaxation rates and mobility are significant at low densities. We also find that at very low temperatures the IFR determines the mobility for high density and at room temperature the polar LO phonon scattering dominates for all concentrations considered. Using the full form for the relaxation rates of acoustic phonons and taking into account many-body effects we obtain a good agreement with recent experiment [16]. 


\section{ACKNOWLEDGEMENT}

This research is funded by Vietnam National Foundation for Science and Technology Development (NAFOSTED) under Grant number 103.01-2017.23.

\section{REFERENCES}

[1] A. Ohtomo et al., Appl. Phys. Lett, 72 (1998) 2466.

[2] T. Aoki et al., Appl. Phys. Lett, 76 (2000) 3257.

[3] U. K. Mishra et al., Proc. IEEE, 90 (2002), 1022.

[4] K. Koike, Jpn. J. Appl. Phys. 43 (2004) L1372.

[5] S. Datta, B. Das, Appl. Phys. Lett. 56 (1990) 665.

[6] K. Tsubaki et al., Appl. Phys. Lett. 80 (2002) 3126.

[7] K. Ellmer, J. Phys. D: Appl. Phys. 34 (2001) 3097.

[8] D. C. Look, Semicond. Sci. Technol. 20 (2005) S55.

[9] A. Gold, Appl. Phys. Lett. 96 (2010) 242111.

[10] A. Gold, J. Appl. Phys. 110 (2011) 043702.

[11] M. Nakano et al., Adv. Mater. 22 (2010) 876.

[12] M. A. Tsukazaki et al., Nature Mater. 9 (2010) 889.

[13] M. Tsaousidou, Phys. Status Solidi RRL 7 (2013) 544.

[14] T. Kawamura, S. Das Sarma, Phys. Rev. B 45 (1992) 3612.

[15] K. Rizwana Begum et al., 2013 AIP Conference Proceedings, Volume 1536, Issue 1, p.447.

[16] J. Falson et al., Appl. Phys. Express 4 (2011) 091101.

[17] Q. Li et al., Appl. Phys. Express 6 (2013) 121102.

[18] Vo Van Tai, Nguyen Quoc Khanh, Physica E 67 (2015) 84.

[19] A. Gold, Z. Phys. B 103 (1997) 491.

[20] A. Gold, Phys. Rev. B 35 (1987) 723.

[21] Chihiro Hamaguchi, Basic Semiconductor Physics, Springer, Berlin Heidelberg, 2001.

[22] A. Gold, Semicond. Sci. Technol. 26 (2011) 045017.

[23] A. Gold, Appl. Phys. Lett. 54 (1989) 2100.

[24] S. J. MacLeod et al., Phys. Rev. B 80 (2009) 035310.

[25] M. D. Kamatagi, et al., Phys. Rev. B 71 (2005) 125334. 